\title{
Time to be Alert!! Stay Safe from the Omicron Variant of COVID-19
}

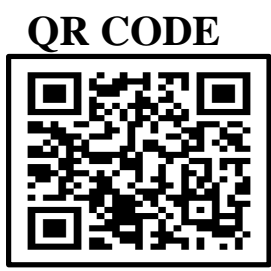

Dr. SAHIL THAKAR

Dear Authors and Readers,

Greetings

At the time of writing this editorial, the new variant of the COVID-19 virus, named "Omicron" has emerged as a threat $t$ the whole world.

Therefore, this editorial is written to all our authors and readers who visit our website and read the scientific articles published by the journal.

It was on $26^{\text {th }}$ November 2021 that WHO designated the variant B.1.1.529, named Omicron, on the advice of WHO's Technical Advisory Group on Virus Evolution (TAG-VE). ${ }^{1}$ This variation was first reported to WHO from South Africa on $24^{\text {th }}$ November 2021. This puts Omicron into the mosttroubling category of Covid-19 variants, along with the globally-dominant Delta, plus its weaker rivals Alpha, Beta and Gamma.

While publishing the manuscript, countries like Australia, Belgium, Botswana, Canada, Denmark, France, Germany, Hong Kong, Israel, Netherlands, Portugal, Scotland, South Africa, Switzerland (probable case), and the UK have all reported cases, while other countries have imposed travel restrictions to prevent the virus from reaching the country. ${ }^{2}$

While there is no clear understandings of the transmission and severity caused by this variant (at the moment), I, on behalf of the entire editorial board request you all to take proper precautions and no to be lax in any manner. Till now we have fought and won against this virus, we again need to show resilience and prevent the spread of this variant until research proves its severity and vaccine effectiveness against this variant.

This would also be the time to remind you all to maintain proper social distancing norms, to always wear a mask and wash/sanitize their hands frequently. You are also advised to try to stay indoors as much as possible and avoid unnecessary travel.

This pandemic has again put the age-old saying of "Its better to be safe than sorry" as a saying of wisdom in a COVID-19 affected world as the cost of laxicity can ultimately result in death, which would not only be a great loss to any family as well as a country, but be a great loss to humanity.

\section{REFERENCES}

1. World Health Organization (WHO). Update on Omicron (Online Article). Available from: https://www.who.int/news/item/28-11-2021-updateon-omicron [Last Accessed on 29th November, 2021] 2. Times of India. (Online Article). https://timesofindia.indiatimes.com/india/coronaviru s-live-updates-india-and-world-november28/liveblog/87955934.cms [Last Accessed on 29th November, 2021] 
Cite this article as:

Thakar S. Time to be Alert!! Stay Safe from the Omicron Variant of COVID-19. Int Healthc

Res J. 2021;5(8):EC1-EC2. https://doi.org/10.26440/IHRJ/0508.11476

\section{AUTHOR AFFILIATIONS:}

Co-Editor, IHRJ \& Associate Professor (Reader), Department of Public Health Dentistry, Himachal Dental College, Sundernagar, H.P., India

e-mail id for correspondence: editor[dot]ihrj[at]ihrjournal[dot]com 